\title{
Rapid Identification of Pathogens from Pediatric Blood Cultures by Use of the FilmArray Blood Culture Identification Panel
}

\author{
Xiaotian Zheng, ${ }^{\text {a,b }}$ Wanda Polanco, ${ }^{\text {a Donna Carter, }}{ }^{\text {a }}$ Stanford Shulman ${ }^{\text {a,b }}$ \\ Ann \& Robert H. Lurie Children's Hospital of Chicago, Chicago, Illinois, USA ${ }^{a}$; Northwestern University Feinberg School of Medicine, Chicago, Illinois, USA
}

\begin{abstract}
The performance of the FilmArray blood culture identification (BCID) panel has been studied in adult patients. We describe here an evaluation of this assay for the rapid identification of pathogens in Bactec Peds Plus/F and Bactec standard anaerobic/F bottles that contained blood samples from pediatric patients at a tertiary care children's hospital.
\end{abstract}

$\mathrm{B}$ loodstream infections are associated with high fatality rates. Rapid organism identification and susceptibility testing may improve patient outcomes $(1,2)$. In 2013, the FDA cleared a rapid and automated multiplex PCR assay, the FilmArray blood culture identification (BCID) (BioFire Diagnostics, Salt Lake City, UT), which directly identifies common pathogens, including 7 genera/ species of Gram-positive bacteria, 10 genera/species of Gram-negative bacteria, and 5 species of Candida (as well as 3 resistance determinants) in the positive blood culture bottles. The assay requires about 2 min of hands-on sample processing time and $1 \mathrm{~h}$ of instrument time (which includes DNA isolation, amplification, and detection), which promises to shorten the pathogen identification time by $\geq 1$ to 2 days.

However, a demonstration of the clinical performance of the assay by the manufacturer was limited to the BD Bactec Plus aerobic/F medium. There are 5 articles reporting the performance of the assay (3-7), but the specimens in these studies were collected mainly from adult patients, and there was very little information regarding the pediatric bottles/media used for testing. There is a paucity of performance data of this assay for the testing of pediatric bottles and pediatric patients. We sought to evaluate the performance of the FilmArray BCID system on pediatric specimens by comparing the results to those of conventional blood culture workup methods, which included a subculture of broth onto solid medium for further pathogen identification and antimicrobial susceptibility testing using phenotypic assays.

(The results of this study were presented in part at the 24th European Congress of Clinical Microbiology and Infectious Diseases in Barcelona, Spain, 10 to 13 May 2014).

The study was conducted during an 8-month period from January to August in 2013. Blood culture specimens were collected from our academic tertiary care pediatric medical center. Each blood culture included a Bactec Peds Plus/F bottle and a Bactec standard anaerobic/F bottle (Becton, Dickinson, Cockeysville, $\mathrm{MD})$. All bottles were incubated in the Bactec FX continuous monitoring blood culture instrument. Prospectively, the FilmArray BCID assay was performed after routine Gram stain examination with the first bottle (Peds Plus/F or standard anaerobic/F bottle) that signaled positive growth. The results of the BCID assay were compared to those obtained using standard bacterial identification and antibiotic susceptibility using the MicroScan system (Siemens, Sacramento, CA). The MicroScan panels used included PC 33 for Gram-positive bacteria, NC 50 for Gram-negative bacteria, and RY-ID for yeast identification. They were prepared and run on the MicroScan WalkAway instrument, according to the manufacturer's instructions. Multiple isolates of an organism from a patient were included in the analysis only once. To resolve discrepancies, matrix-assisted laser desorption-ionization time of flight mass spectrometry (MALDI-TOF MS) and 16S rRNA gene sequencing were performed. For MALDI-TOF MS, both the FDAcleared version (clinical application $[\mathrm{CA}]$ ) and the research use only (RUO) version were used, according to the manufacturer's instructions (Bruker Daltonics, Billerica, MA). The 16S rRNA gene sequencing was performed using the MicroSeq $50016 \mathrm{~S}$ rRNA gene bacterial identification system (Life Technologies, Foster City, CA). A public database, GenBank, was used for sequence analysis. To evaluate the BCID assay using archived bacteria, blood culture bottles were spiked with a bacterial suspension containing 100 to $1,000 \mathrm{CFU}$ of each organism. These bottles were incubated in the Bactec FX instrument until the instrument signaled positive growth, and the broths were subsequently processed in the same way as actual patient blood cultures.

After excluding duplicates, the results for 166 positive blood cultures collected from 138 different children were included for data analysis.

Overall, the FilmArray BCID assay identified 168 (89.4\%) of the 188 organisms recovered by culture. For the 20 organisms that the BCID assay failed to detect, 13 (65\%) were organisms that the FilmArray BCID assay was not designed to detect.

As shown in Table 1, culture yielded 122 Gram-positive bacteria. The BCID assay identified $112(91.8 \%)$ organisms. Of the 116 organisms the BCID assay is designed to detect, 112 (96.6\%) were correctly identified by the assay. These included Streptococcus pyogenes (group A streptococcus [GAS]) (3/3 detected), Streptococcus agalactiae (group B streptococcus [GBS]) (2/2 detected), Streptococcus pneumoniae (1/1 detected), non-pneumoniae Streptococcus (8/9 detected), Staphylococcus aureus (31/31 detected), coagulasenegative staphylococci (55/57 detected), and enterococci (12/13 detected). The BCID assay missed identifying the following 4 bacteria: Staphylococcus hominis, a nonviable Gram-positive coccus in

\footnotetext{
Received 24 July 2014 Returned for modification 31 July 2014 Accepted 25 September 2014

Published ahead of print 1 October 2014

Editor: K. C. Carroll

Address correspondence to Xiaotian Zheng,x-zheng@northwestern.edu Copyright $\odot$ 2014, American Society for Microbiology. All Rights Reserved. doi:10.1128/JCM.02133-14
} 
TABLE 1 Pathogen detection results

\begin{tabular}{|c|c|c|c|c|c|}
\hline Pathogen & $\begin{array}{l}\text { No. recovered } \\
\text { by culture }\end{array}$ & $\begin{array}{l}\text { No. (\%) identified } \\
\text { by BCID assay }\end{array}$ & \multicolumn{2}{|c|}{ No. of organisms recovered by culture that are: } & $\begin{array}{l}\% \text { recovery of organisms } \\
\text { included in the BCID } \\
\text { assay }\end{array}$ \\
\hline Gram-positive bacteria & 122 & $112(91.8)$ & 6 & 4 & 96.6 \\
\hline Gram-negative bacteria & 58 & $50(86.2)$ & 6 & 2 & 96.2 \\
\hline Candida & 8 & $6(75)$ & 1 & 1 & 85.7 \\
\hline
\end{tabular}

clusters, Enterococcus faecium (in a mixed culture with a Gramnegative rod, which was correctly identified), and a viridans streptococcus (mixed with Staphylococcus capitis, which was correctly identified as a coagulase-negative staphylococcus). The BCID assay did not identify the following 6 bacteria for which specific primers are not included in the design of the assay: Peptostreptococcus spp., Rothia mucilaginosa, Corynebacterium spp., Bacillus spp., Staphylococcus pettenkoferi, and Staphylococcus saccharolyticus (this bacterium can be detected but at a much lower sensitivity, as described in the product insert). The oxacillin resistance determinant mecA was correctly identified for all 86 Staphylococcus spp., including 6 methicillin-resistant S. aureus (MRSA) organisms, 25 methicillin-susceptible S. aureus (MSSA) organisms, 37 oxacillin-resistant coagulase-negative staphylococci, and 18 susceptible coagulase-negative staphylococci. Compared to the susceptibility results, the BCID assay correctly reported 1 positive and 11 negative $v a n A$ or $v a n B$ results for the enterococci.

For the Gram-negative bacteria, the FilmArray BCID assay correctly identified 50 of 58 organisms (86.2\%). Fifty-two of the 58 were the organisms the BCID assay was designed to detect, and the assay actually detected $96.2 \%$ (50 out of 52) of these. These correct identifications for organisms at either the genus or species levels included 14 Escherichia coli, 13 Klebsiella pneumoniae, 9 Enterobacter cloacae, 4 Pseudomonas aeruginosa, 4 Serratia marcescens, 1 Acinetobacter baumannii, 1 Haemophilus influenzae, 1 Proteus mirabilis (the BCID result was Proteus), 1 Citrobacter freundii, 1 Salmonella sp., and 1 Pantoea agglomerans (the BCID result for the last three organisms was Enterobacteriaceae). Of two cultures growing P. agglomerans and identified by MicroScan, the BCID assay was negative for 1 and inaccurately reported $S$. marcescens in the other. According to manufacturer's product insert, BCID assay identifies Pantoea spp. with reduced sensitivity. There is no description regarding cross-reactivity with $S$. marcescens. These two particular isolates lost viability after storage and could not be tested with other methodologies. To investigate this possibility of cross-reactivity, we tested a batch of Pantoea spp. that included 1 ATCC strain, 1 College of American Pathologists (CAP) proficiency sample, and 2 archived clinical isolates collected from previous patients. As shown in Table 2, the BCID assay missed identifying the ATCC stain and misidentified the others as S. marcescens. In the entire study, this was the only organism misidentification found.

The other 6 Gram-negative bacteria that the BCID assay did not identify were organisms that the FilmArray assay was not designed to detect: Stenotrophomonas maltophilia (2 organisms), Capnocytophaga spp., Prevotella melaninogenica, Haemophilus parainfluenzae, and Hafnia alvei.

The BCID assay correctly identified 6 of 8 positive blood cultures growing Candida species. These included C. glabrata, C. parapsilosis, C. tropicalis, and C. albicans. It did not identify a sample containing Candida lusitaniae, which the FilmArray BCID assay was not designed to detect. The BCID assay also missed detecting C. glabrata present in a mixed blood culture with Enterococcus, which was correctly identified by this assay.

Twenty blood cultures grew more than one organism. Among them, two grew bacteria that the BCID assay was not designed to detect $(H$. parainfluenzae and $H$. alvei). For the rest of the 18 mixed cultures, the BCID assay correctly identified 9 (50\%). The BCID assay missed the identification of 3 organisms in 3 mixed cultures. These included a viridans streptococcus, a C. glabrata, and an E. faecium culture. The BCID assay identification results were inconclusive for the remaining 6 mixed cultures because the two organisms growing in the same bottle shared the same genus/ group, and at least one of the two organisms was a species that the BCID assay was not designed to detect.

Recent studies have demonstrated that the rapid detection of bloodstream pathogens benefits patient care $(1,2)$. Working closely with the antimicrobial stewardship teams, the implemen-

TABLE 2 Identification of Pantoea spp. using BCID assay and other reference methods

\begin{tabular}{|c|c|c|c|c|c|}
\hline Pantoea organism & $\begin{array}{l}\text { MicroScan } \\
\text { results }\end{array}$ & $\begin{array}{l}\text { MALDI-TOF } \\
\text { MS, CA database }\end{array}$ & $\begin{array}{l}\text { Result of MALDI- } \\
\text { TOF MS, RUO } \\
\text { database (scores) }\end{array}$ & $\begin{array}{l}\text { 16S rRNA gene } \\
\text { sequencing result }\end{array}$ & FilmArray BCID assay result ${ }^{a}$ \\
\hline ATCC 29002 & P. agglomerans & No identification & P. dispersa (2.124) & P. agglomerans/P. dispersa & Not detected, not detected \\
\hline CAP 2012 (D-15) & P. agglomerans & No identification & P. septica (2.408) & P. septica & $\begin{array}{l}\text { Enterobacteriaceae, Enterobacteriaceael } \\
\text { S. marcescens }\end{array}$ \\
\hline $\begin{array}{l}\text { Archived clinical isolate } \\
\quad 1 \text { (blood) }\end{array}$ & P. agglomerans & No identification & P. calida (1.877) & P. calida/P. gaviniae & $\begin{array}{l}\text { Enterobacteriaceae/S. marcescens, } \\
\text { Enterobacteriaceae/S. marcescens }\end{array}$ \\
\hline $\begin{array}{l}\text { Archived clinical isolate } \\
\quad 2 \text { (tissue) }\end{array}$ & P. agglomerans & No identification & P. calida (2.357) & P. calida/P. gaviniae & $\begin{array}{l}\text { Enterobacteriaceae/S. marcescens, } \\
\text { Enterobacteriaceae/S. marcescens }\end{array}$ \\
\hline
\end{tabular}

${ }^{a}$ Results are for the Peds Plus/F and standard anaerobic/F bottles, respectively. 
tation of rapid molecular or mass spectrometry-based pathogen identification systems has been shown to decrease the time to appropriate and optimal antimicrobial therapy, lower mortality, shorten hospital stays, and reduce overall health care costs for these patients $(1,2,8)$ MALDI-TOF MS has been shown to be an effective and reliable method for the rapid identification of pathogens in broth from blood culture bottles (1). It is fast, capable of detecting a wide range of pathogens, and cost-effective, although it requires manual processing, such as centrifugation or filtration concentration. The method generally does not report susceptibility results. The MALDI-TOF MS procedures for blood culture are still being standardized. Another available platform is the automated Verigene blood culture system. Designed as separate assays, Verigene uses a microarray format to detect Gram-positive pathogens (BC-GP) (detection time, $2.5 \mathrm{~h}$ ) and Gram-negative pathogens (BC-GN) (detection time $2 \mathrm{~h}$ ) separately. Uniquely, the BC-GN assay detects some important antimicrobial resistance determinants, including $K$. pneumoniae carbapenemase (KPC), NDM, VIM, IMP, and OXA for carbapenems and CTX-M for extended-spectrum $\beta$-lactamases (ESBL). Evaluations have shown clinical utility for this system (8-12). In comparison, the FilmArray BCID assay is a combined panel. Five studies report the performance of this assay with clinical specimens (3-7). Blaschke et al. (3) reported a comparison of a developmental version of this assay with standard culture and susceptibility methods and found a $91 \%$ detection rate for the pathogens included in the panel by design. In that study, 102 blood culture samples were collected at a pediatric medical center and a clinical reference laboratory for testing, but there are no details about the bottle/medium types used or the number of samples/pathogens collected from children and from adult patients. From a Swedish university hospital, Altun et al. (4) reported a $91.6 \%$ identification rate of the positive blood culture bottles that grew single organisms and a $71 \%$ identification rate for polymicrobial bottles. Among the total of 167 bottles growing single pathogens, 9 were BacT/Alert PF Plus pediatric bottles, while the rest were collected in 3 other bottle types. The four bottle types studied were found to perform equally well. In another study reported by Rand and Delano (5), 161 blood cultures were collected in BD Bactec plus aerobic/F bottles. There was no description about whether pediatric samples were included. For the blood culture bottles that grew single pathogens, the BCID assay identified 98\% (48/49) of those organisms that would be expected to be identified only to the genus level. The BCID assay also identified all of the other 84 pathogens (100\%) to the species/complex levels. Most recently, Bhatti et al. (6) reported a comparison of the performances of the BCID and the Verigene systems using the Bactec culture bottles. However, no bottle/medium type or patient population type (pediatric versus adult) was specified. To date, there is no report in the literature documenting the evaluation of BCID testing specifically on pediatric media collected from children. The results of our present study demonstrate the accuracy of the assay in this specific setting. The advantages of the assay include only 2 to 3 min of hands-on processing and $1 \mathrm{~h}$ of instrument time. The ease of use may help laboratory adaptation of the assay to be performed during both day and night shifts.

One limitation of the present study is the lack of testing of certain pathogens. During the study period, Listeria monocytogenes and Neisseria meningitidis were not encountered. Interestingly, Pardo et al. (13) recently reported a child in whom N. meningitidis was detected successfully from blood and cerebrospinal fluid (CSF) cultures using the BCID assay. No KPC-producing organism was found in our study, and there was only 1 case described (6) in previous reports evaluating this assay. In our laboratory, a KPC-positive K. pneumoniae isolate was spiked into a blood culture bottle and correctly identified by the BCID assay.

In conclusion, the FilmArray BCID assay performed very well for the rapid identification of pathogens in positive blood culture bottles that contained pediatric medium and blood samples from children. Compared to standard phenotypic methods, the assay reduced the time for pathogen identification by 1 to 2 days. The ease of use of the assay will likely help laboratories adapt and offer it 24 hours a day, 7 days a week, thus improving patient care.

\section{ACKNOWLEDGMENT}

We thank BioFire Diagnostics for providing the BCID assay kits used for the study.

\section{REFERENCES}

1. Huang AM, Newton D, Kunapuli A, Gandhi TN, Washer LL, Isip J, Collins CD, Nagel JL. 2013. Impact of rapid organism identification via matrix-assisted laser desorption/ionization time-of-flight combined with antimicrobial stewardship team intervention in adult patients with bacteremia and candidemia. Clin. Infect. Dis. 57:1237-1245. http://dx.doi.org /10.1093/cid/cit498.

2. Perez KK, Olsen RJ, Musick WL, Cernoch PL, Davis JR, Land GA, Peterson LE, Musser JM. 2013. Integrating rapid pathogen identification and antimicrobial stewardship significantly decreases hospital costs. Arch. Pathol. Lab. Med. 137:1247-1254. http://dx.doi.org/10.5858/arpa.2012 -0651-OA.

3. Blaschke AJ, Heyrend C, Byington CL, Fisher MA, Barker E, Garrone NF, Thatcher SA, Pavia AT, Barney T, Alger GD, Daly JA, Ririe KM, Ota I, Poritz MA. 2012. Rapid identification of pathogens from positive blood cultures by multiplex polymerase chain reaction using the FilmArray system. Diagn. Microbiol. Infect. Dis. 74:349-355. http://dx.doi.org /10.1016/j.diagmicrobio.2012.08.013.

4. Altun O, Almuhayawi M, Ullberg M, Ozenci V. 2013. Clinical evaluation of the FilmArray blood culture identification panel in identification of bacteria and yeasts from positive blood culture. J. Clin. Microbiol. 51: 4130-4136. http://dx.doi.org/10.1128/JCM.01835-13.

5. Rand KH, Delano JP. 2014. Direct identification of bacteria in positive blood cultures: comparison of two rapid methods, FilmArray and mass spectrometry. Diagn. Microbiol. Infect. Dis. 79:293-297. http://dx.doi.org /10.1016/j.diagmicrobio.2014.03.014.

6. Bhatti MM, Boonlayangoor S, Beavis KG, Tesic V. 2014. Evaluation of FilmArray and Verigene systms for rapid identification of positive blood cultures. J. Clin. Microbiol. 529:3433-3436. http://dx.doi.org/10.1128 /JCM.01417-14.

7. Almuhayawi M, Altun O, Strålin K, Ozenci V. 2014. Identification of microorganisms by FilmArray and matrix-assisted laser desorption ionization-time of flight mass spectrometry prior to positivity in the blood culture system. J. Clin. Microbiol. 52:3230-3236. http://dx.doi.org/10 .1128/JCM.01084-14.

8. Sango A, McCarter YS, Johnson D, Ferreira J, Guzman N, Jankowski CA. 2013. Stewardship approach for optimizing antimicrobial therapy through use of a rapid microarray assay on blood cultures positive for Enterococcus species. J. Clin. Microbiol. 51:4008-4011. http://dx.doi.org /10.1128/JCM.01951-13.

9. Wojewoda CM, Sercia L, Navas M, Tuohy M, Wilson D, Hall GS, Procop GW, Sandra S, Richter SS. 2013. Evaluation of the Verigene Gram-positive blood culture nucleic acid test for rapid detection of bacteria and resistance determinants. J. Clin. Microbiol. 51:2072-2076. http: //dx.doi.org/10.1128/JCM.00831-13.

10. Samuel LP, Tibbetts RJ, Agotesku A, Fey M, Hensley R, Meier FA. 2013. Evaluation of a microarray-based assay for rapid identification of Grampositive organisms and resistance markers in positive blood cultures. J. Clin. Microbiol. 51:1188-1192. http://dx.doi.org/10.1128/JCM.02982-12.

11. Tojo M, Takahiro Fujita T, Ainoda Y, Nagamatsu M, Hayakawa K, Mezaki K, Sakurai A, Masui Y, Yazaki H, Takahashi H, MiyoshiAkiyama T, Totsuka K, Kirikae T, Ohmagari N. 2014. Evaluation of an 
automated rapid diagnostic assay for detection of Gram-negative bacteria and their drug resistance genes in positive blood cultures. PLoS One 9:e94064. http://dx.doi.org/10.1371/journal.pone.0094064.

12. Sullivan KV, Deburger B, Roundtree SS, Ventrola CA, Blecker-Shelly DL, Mortensen JE. 2014. Pediatric multicenter evaluation of the Verigene Gram-negative blood culture test for rapid detection of inpatient bacteremia involving Gram-negative organisms, extended-spectrum beta- lactamases, and carbapenemases. J. Clin. Microbiol. 52:2416-2421. http: //dx.doi.org/10.1128/JCM.00737-14.

13. Pardo J, Klinker KP, Borgert SJ, Butler BM, Rand KH, Iovine NM. 2014. Detection of Neisseria meningitidis from negative blood cultures and cerebrospinal fluid with the FilmArray blood culture identification panel. J. Clin. Microbiol. 52:2262-2264. http://dx.doi.org/10.1128/JCM $.00352-14$. 